\title{
A multichannel system for recording and analysis of cortical field potentials in freely moving rats
}

\author{
Fu-Zen Shaw a , Ruei-Feng Chen ${ }^{\mathrm{b}}$, Hen-Wai Tsao ${ }^{\mathrm{a}}$, Chen-Tung Yen ${ }^{\mathrm{b}, *}$ \\ a Department of Electrical Engineering, National Taiwan University, Roosevelt Rd., Taipei, Taiwan, ROC \\ ${ }^{\mathrm{b}}$ Department of Zoology, National Taiwan University, Section 4, Roosevelt Rd., Taipei, Taiwan, ROC
}

Received 28 May 1998; received in revised form 13 October 1998; accepted 16 December 1998

\begin{abstract}
A system has been developed to record and analyze the cortical electrical activity from 16 different sites in freely moving rats. The hardware includes a 16-channel amplifier system whose high input impedance, low noise, small size, light weight and shielded multistrand connecting cable allow high quality multichannel recording of field potentials. The software developed for this system consists of data acquisition, data analysis and topographic mapping of cortical-evoked potentials as well as electroencephalograms. Cortical field potentials evoked by $\mathrm{CO}_{2}$-laser stimulation were compared between wakeful and pentobarbital-treated conditions. To investigate the background interference produced by sleep spindle, three kinds of reference-free methods (the Wilson, local average and weighted average methods) were utilized to compare the coherence between field potentials obtained from two cerebral hemispheres using monopolar vs. reference-free recordings. (c) 1999 Elsevier Science B.V. All rights reserved.
\end{abstract}

Keywords: Chronic recording; Amplifier; Evoked potential; EEG; Nociception

\section{Introduction}

Early in this century, Head and Holmes (1911) observed that the threshold for pain stimuli is preserved after damage to cerebral cortex, and the pain threshold and pain sensation severely changes with damage to the thalamus in patients. These observations have led to the still widely held view that pain is sensed in the thalamus. Over the past two decades, however, the results from human (Bromm and Treede, 1987; Jones et al., 1991; Kenshalo and Willis, 1991; Talbot et al., 1991; Kitamura et al., 1995), primate (Kenshalo et al., 1988; Dong et al., 1989), cat (Matsumoto et al., 1988), and rat experiments (Lamour et al., 1983a,b; Schouenborg et al., 1986; Kalliomaki et al., 1993; Yen et al., 1994), have indicated that the cerebral cortex participates in

\footnotetext{
* Corresponding author. Tel.: + 886-2-23630231, extn. 3322; fax: $+886-2-23636837$.

E-mail address: ctyen@ccms.ntu.edu.tw (C.-T. Yen)
}

the processing of pain. Positron emission tomography (PET) or functional magnetic resonance imaging (fMRI) studies in human subjects (Jones et al., 1991; Talbot et al., 1991) have revealed that many areas of the cerebral cortex, such as primary somatosensory cortex (SI), secondary somatosensory cortex (SII) and cingulate gyrus, are activated by pain stimuli. The activated areas of the cerebral cortex have been described using unit recordings and field potentials studied in anesthetized monkeys (Kenshalo and Isensee, 1983; Kenshalo and Willis, 1991), cats (Matsumoto et al., 1988), and rats (Lamour et al., 1983a,b; Schouenborg et al., 1986; Kalliomaki et al., 1993). However, activated areas were not clearly described in wakeful animals because the nociceptive signal is strongly diminished by anesthetics (Kochs et al., 1990; Vogt, 1991). Moreover, the temporal consequence of the pain processing in cerebral cortex has not been well investigated. By simultaneously recording neuronal signals from various cortical areas, we may gain a deeper 
understanding of the cortical areas involved with processing nociceptive stimuli. It will also be possible to investigate the temporal relationships of this processing among different cortical areas. Therefore, we developed a multichannel recording and analysis system to record and analyze the signals in various cortical areas evoked by different stimuli, i.e. electrical, mechanical and $\mathrm{CO}_{2}$ laser stimulation, in conscious and/or anesthetized rats.

This paper describes a general-purpose system for amplification, acquisition, storage and analysis of signals arising from different cortical recording sites. This low noise and light-weight multichannel system is capable of collecting signals from 1 to 16 channels. An on-line interactive icon-based analytic program provides temporal, spectral and spatial analysis of these signals. Moreover, reference-free derivation is developed for multichannel cortical surface signal analysis. We can supply the program free of charge upon request if the e-mail address of the requester is available and if three 3.5 in. floppy disks and the return postage are provided.

\section{Materials and methods}

\subsection{General surgical procedures}

Male Wistar rats (250-300 g) were anesthetized with sodium pentobarbital (50 mg/kg, i.p.) initially. Ketamine was administered as necessary to maintain anesthesia. Once anesthesia was induced, the dorsal surface of the animal's head was shaved and the rat was placed in a stereotaxic apparatus. A midline scalp incision was made. Connective tissue was scraped away from the skull surface. The skull was roughened with a bone curette and then cleaned with saline. After exposure of the skull, a small concavity on each marked position of the skull was drilled first and a stainless steel screw was securely driven into this position. The small screw electrodes ( $1 \mathrm{~mm}$ in diameter) were symmetrically arranged on both sides of the head from anterior to posterior separated by $3 \mathrm{~mm}$. The precise location of the recording channels is shown in the inset of Fig. 3. These electrodes were placed to avoid penetration of the underlying aura. Monopolar recordings were made from these screws. The screw used as the reference electrode was implanted $2 \mathrm{~mm}$ caudal to lambda. Each individual screw was connected to a pin in a $2 \times 2$ male connector module $\left(25 \mathrm{~mm}^{2}\right)$. To assure proper function of electrodes after surgery, evoked potentials (EPs) were elicited by stimulating the tail of the rat using a low intensity $(0.5 \mathrm{~ms}, 0.1 \mathrm{~mA})$, constant electric current pulse (Grass S44). Subjects without identifiable EPs at leads 5 or 9 , which overlie the tail representative area of the primary somatosensory cortex (Chapin and Lin, 1990), were eliminated from the study. Dental cement was then applied to the skull screws, skull surface and connector modules in order to secure the connector in place and to fasten it to the surface of the skull. The rats were housed individually in cages, $46 \times 24 \times 21 \mathrm{~cm}$ in length, width and height, respectively. Animals were then injected with antibiotics (Combion-S, $60000 \mathrm{U} / \mathrm{kg}$ i.p.).

\section{2. $\mathrm{CO}_{2}$ laser stimulator and recording protocol}

The skin was stimulated with radiant heat pulses emitted from a $\mathrm{CO}_{2}$ laser (medical surgical laser, Tjing Ling \# 2, National Taiwan University, $10.6 \mu \mathrm{m}$ wavelength) operating in $\mathrm{TEM}_{00}$ mode (Gaussian distribution) (Yen et al., 1994). The beam diameter was $3 \mathrm{~mm}$ (unfocused). The duration of the stimulation pulse was kept at $10 \mathrm{~ms}$. The output power was between 6 and 18 W. These output energies corresponded to $60-180 \mathrm{~mJ}$. These stimulation energies did not result in any visible damage to the skin. In order to minimize tissue damage, sensitization and habituation, the stimuli were randomly applied to a local skin area $(1 \mathrm{~cm}$ for tail base and middle tail; $1.5 \mathrm{~cm}$ for tail tip). The interstimulus interval was longer than $10 \mathrm{~s}$.

One month following recovery from surgery, animals were placed in an acrylic recording chamber with a slit on the top. This chamber was designed to allow recording from unrestrained animals. The behavior of the rat could be clearly observed. We used a custom-fabricated multistrand printer cable line to connect the connector modules to the scalp of the rat and the self-designed amplifiers (see Section 2.3 for detail) through a slit on the top cover of the chamber. To habituate the rat, it was put in the recording chamber five times $(2 \mathrm{~h}$ per day) before each experiment. The animals were placed in the recording chamber for $30 \mathrm{~min}$ prior to the recording session. Surgical and recording procedures complied with NIH (USA) recommended procedures for animal use and care.

\subsection{Multichannel analog amplifier system}

To minimize the size of the recording apparatus, we developed a 16-channel amplifier system. The gain of this amplifier is adjustable with four gain settings $(500 \times, 1000 \times, 2000 \times, 5000 \times)$. The circuit diagram of a single channel of this amplifier is shown in the upper part of Fig. 1. The first stage used an instrumentation amplifier as a pre-amplifier, followed by operational amplifiers with additional gain and bandpass filters. This amplifier system was designed for optimal performance in the recording of cortically derived signals. The presence of both low-level and large commonmode cortical signals necessitated a good common-mode rejection ratio (CMRR) (larger than $100 \mathrm{~dB}$ ) for differential amplifier stage. These signals 


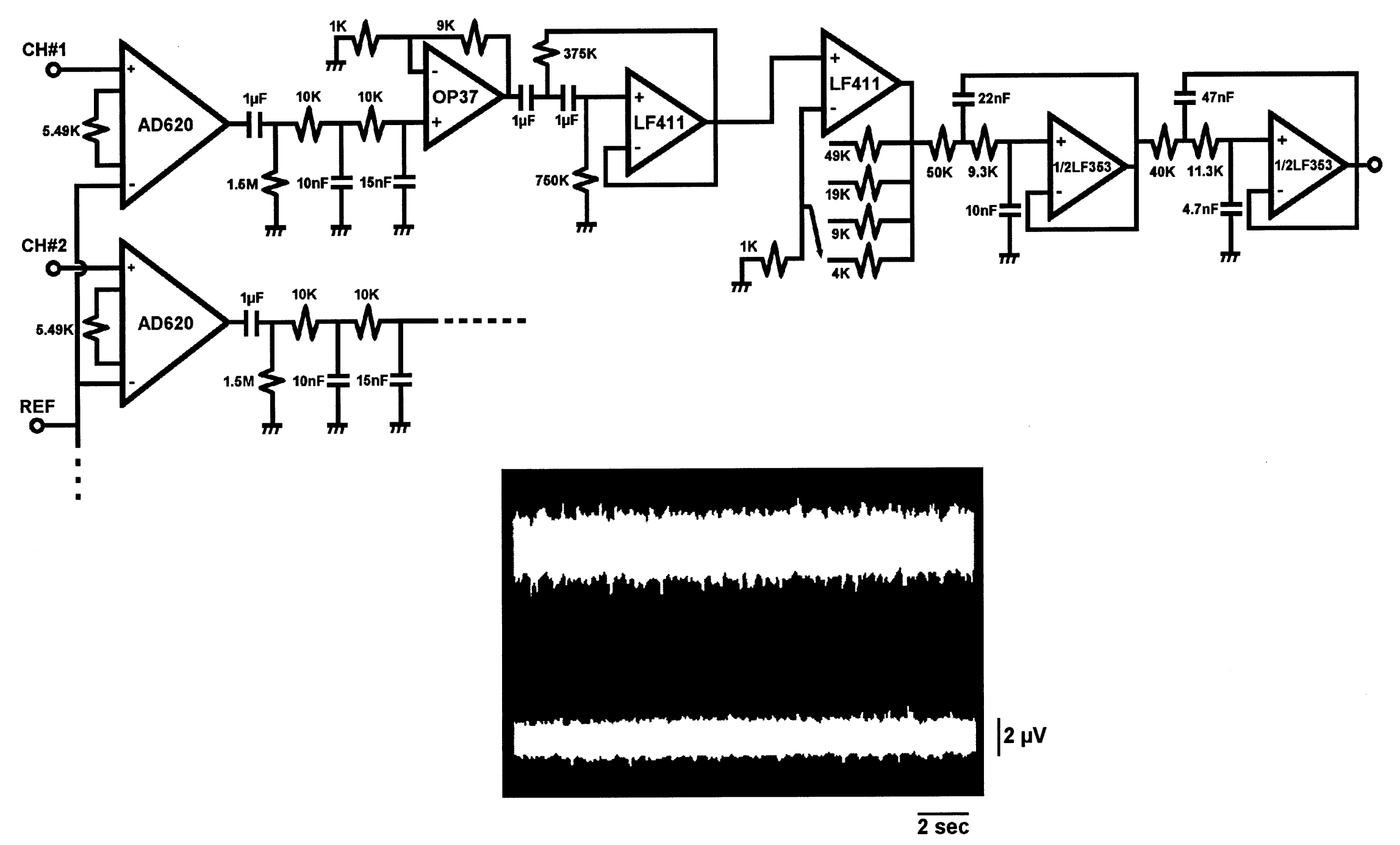

Fig. 1. The circuit diagram and noise of the multichannel amplifier. The noises of our self-designed amplifier $(0.3-500 \mathrm{~Hz})$ and $\mathrm{Grass}$ P511 amplifier $(0.3-300 \mathrm{~Hz})$ were compared. The noise of our self-designed amplifier (lower trace) is about $1.5 \mu \mathrm{V}_{\mathrm{p}-\mathrm{p}}$ and about one-third of that of the Grass amplifier (upper trace). 
also demanded a low noise component in the frequency range of interest, a high input impedance ( $>1 \mathrm{G} \Omega$ ), and high voltage gain $\left(10^{3}-10^{4}\right)$. The first stage was the most critical, so an instrumentation amplifier AD620AN (Analog Devices, PMI Division) was chosen to meet the specifications. It represents a good compromise between performance and cost (unit price around US\$5). The input current noise of AD620AN is 10 $\mathrm{pA}_{\mathrm{p}-\mathrm{p}}$, the measured input voltage noise density is typically $9 \mathrm{nV} / \sqrt{\mathrm{Hz}}$, and the CMRR is $110 \mathrm{~dB}$ with a gain of 10. The gain of the preamplifier is kept low to prevent saturation from DC signals and is held to within $0.1 \%$ tolerance across the entire array in order to maintain a tight match between the signal and reference pre-amplifier. The output of the pre-amplifier passes through the bandpass filter with wider bandwidth, then connects to a low-noise operational amplifier OP37 (Analog Devices, PMI Division), whose voltage noise is $80 \mathrm{nV}_{\mathrm{p}-\mathrm{p}}$, with an additional gain of 10 . The second stage was designed to have a second-order high-pass filter (cutoff frequency, $0.3 \mathrm{~Hz}$ ), four different amplifications and fourth-order low-pass filter (cutoff frequency, $500 \mathrm{~Hz}$ ) with LF411 and LF353 operational amplifiers (National Semiconductor, Santa Clara, CA). To reduce the sampling frequency, a higher order lowpass Butterworth filter design was used. The specifications of the device were satisfactory for evoked potential recordings (Luders et al., 1985). Fourth-order low-pass filters (cutoff frequency, $70 \mathrm{~Hz}$ ) were also built for EEG recording. The DC power source of the operational amplifiers must be bypassed using a capacitor to reduce the interference, especially in the front-end stages. The 16 channels of this amplifier were enclosed in a shielded metal cage, $30 \times 20 \times 8 \mathrm{~cm}$ in length, width and height, respectively. The DC power supply was constructed by general purpose regulators (LM7808 and LM7908, TO-3 package (National Semiconductor, Santa Clara, CA)) with larger capacitors and this was located in a separate shielded enclosure adjacent to the amplifier assembly to reduce interference from the AC power line. The total circuit assembly was laid out on a single sided printed circuit board. In the implementation of this custom amplifier and filter, signal line shielding, an accurate layout and proper grounding was found to be important.

\subsection{Hardware and software specifications}

A PC-486DX66 with $16 \mathrm{Mb}$ of random access memory (RAM) and $540 \mathrm{Mb}$ of hard disk capacity was equipped with a general-purpose data acquisition card (AT-16-MIO-F5, National Instruments, Austin, TX). The AT-16-MIO-F5 board has a fast (up to $200 \mathrm{kHz}$ sample rate) 12-bit ADC with 16 single-ended analogue input channels. This met our specifications for multichannel spatio-temporal analysis. The system was pro- grammed in an icon-based LabVIEW 3.0 software development environment. The trigger signal from the stimulator was connected to the EXTTRIG pin to perform time-lock processing, such as for evoked potentials. The on-line and real-time data-acquisition program, modified from examples of LabVIEW, could display 16-channel EEG/EP waveforms and storage data to hard disk through dual DMA channels. The anti-aliasing sampling frequencies for EP and EEG were 2000 and $250 \mathrm{~Hz}$ respectively. Acquired data were then moved to a $230 \mathrm{Mb}$ magneto-optical disk (Fujitsu) for further temporal, frequency and/or spatial analysis. The analysis programs were also developed with LabVIEW 3.0.

The essential steps used in this program for analyzing evoked field potentials are described below:

1. Recording of EEG-EP epochs: a segment of EEG activity preceding and following the stimulus was digitized and stored on computer disk memory. This operation was repeated about 100-200 times.

2. Selective averaging of EPs: the stored raw single EEG-EP epochs were selected according to specific criteria, and then averaged. EEG-EP segments contaminated by movement artifacts, larger baseline drift, spindle rhythms, and high frequency artifacts induced by chewing action, were eliminated.

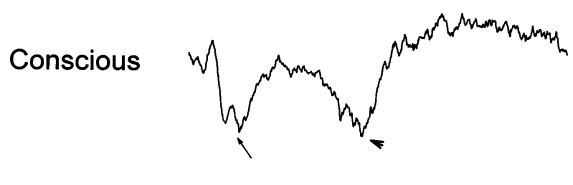

$60 \mathrm{~min}$

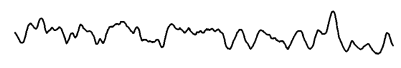

$120 \mathrm{~min}$

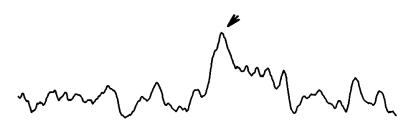

$180 \mathrm{~min}$

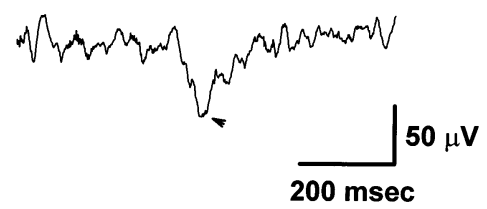

Fig. 2. Cortical evoked potential induced by $\mathrm{CO}_{2}$-laser stimulation under conscious and anesthetized conditions. The middle tail of the rat was stimulated by $12 \mathrm{~W} \mathrm{CO}_{2}$-laser pulses of $10 \mathrm{~ms}$ duration. Two distinct peaks, marked by arrow and arrowhead respectively, were present in the conscious condition. Sodium pentobarbital (i.p., 50 $\mathrm{mg} / \mathrm{kg}$ ) was administered. One hour later, all peaks disappeared. The reversed polarity was present after $2 \mathrm{~h}$ (marked by arrowhead). Three hours later, the peak was restored (marked by arrowhead). These data traces were the averaged responses of channel 5 recordings (as indicated in the inset of Fig. 3). Data traces were selected for averaging, consisting of about 50 stimuli for each trace illustrated. 


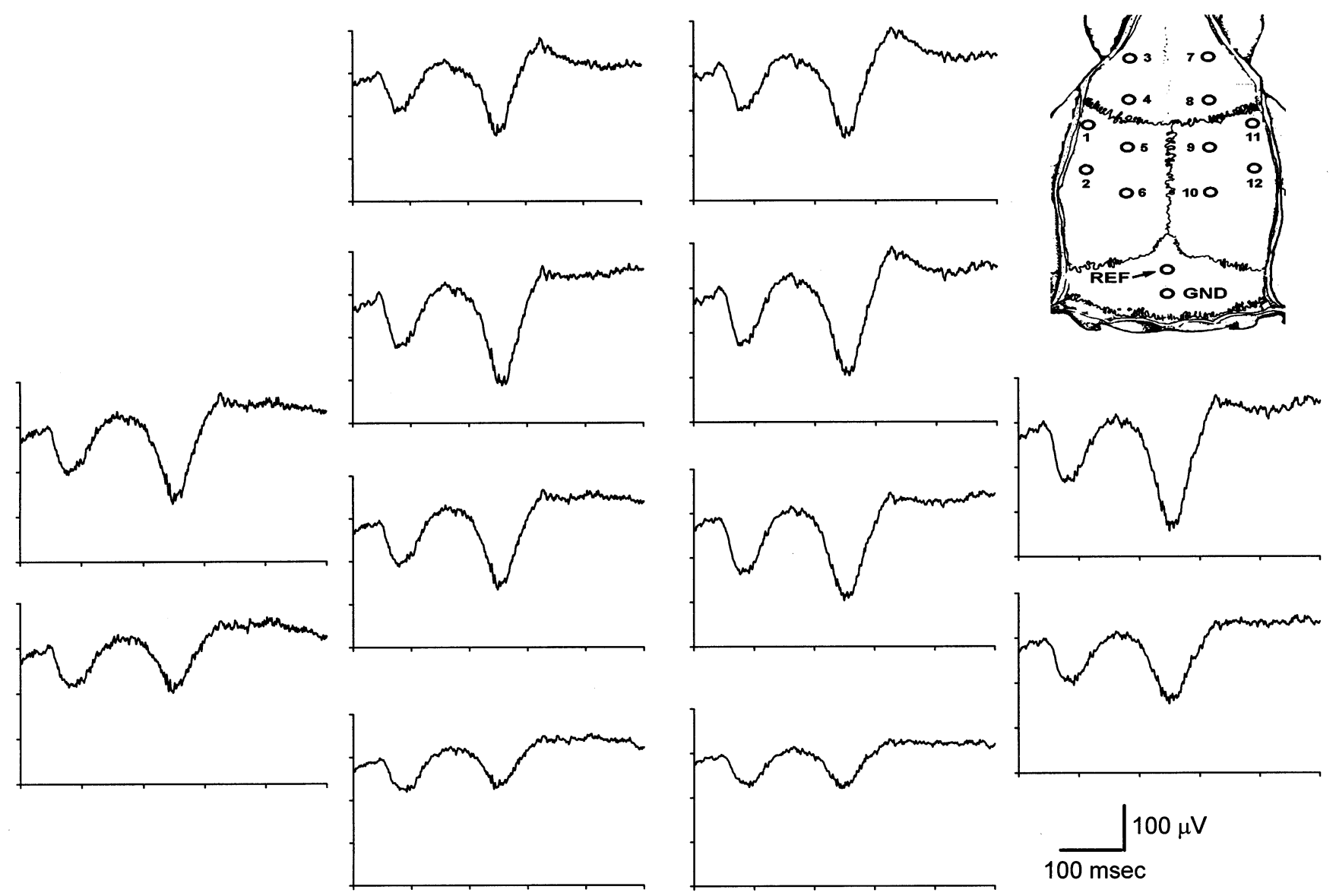

Fig. 3. Spatial distribution of laser-evoked potentials. An example of 12-channel recordings of laser-evoked potentials (12 W at tail base) is displayed in relative recording sites over the skull (indicated in the inset). Each trace represents the average of more than 50 trials.

3. Reference-lead selection: unipolar recordings (lambda reference) were made initially. We could choose whether the selected raw EEG-EP epochs were transferred into reference-free condition or not. Three reference-free methods (Wilson, weighted average and local average methods) were developed here (see detailed description below).

4. Digital filtering: the characteristics of the EP waveform were estimated using a finite impulse response filter with different cutoff frequency settings. The frequency components of EPs were computed using digital filters with zero phase shift (Oppenheim and Schafer, 1989). This allows us to evaluate EP variations in the time and frequency domains.

5. Topographic mapping: the spatial distribution of EP amplitude across the 16 channels was on-line presented on a square plane with grey level coding. Interpolation in this plane was made by summing 16 weighted values, which were calculated from the inverse of the distance between the location and 16 electrodes with the constraint of unitary sum (Perrin et al., 1987). This mode of representation effectively depicted the spatial correlation between recording channels.
The essential steps of the analytic program for EEG are described as follows:

1. Overview original signal: the EEG signal was displayed to mark the contaminated segment. We compared the EEG signal with the observed behavior pattern to reject the contaminated segments. This initial observation of the EEG was also important for subsequent steps, such as reference-lead selection.

2. Reference-lead selection: the same description as in the upper paragraph.

3. Temporal analysis: two kinds of analytical tools were established here. First, the statistical moments (mean, variance, skewness and kurtosis) were used to evaluate the statistical distribution of the magnitude of the selected segments. Moreover, nonlinear characteristics (complexity and phase portrait) of the data segments were established.

4. Frequency analysis: selected EEG segments were transferred into the frequency domain using the fast Fourier transform (FFT) with a Hanning window. The meaningful and well-defined frequency bands (alpha, beta, theta, delta bands) were identified from the EEG spectrum. Coherence was developed to 
evaluate the relationship between two cortical channels.

5. Topographic mapping: the spatial distribution of the temporal/spectral characteristics of the EEG signals in 16 channels was presented on a square

plane with grey level coding. The interpolation on the plane was made by summing 16 weighted values, which were calculated from the inverse of the distance between the location and 16 electrodes with the constraint of unitary sum.

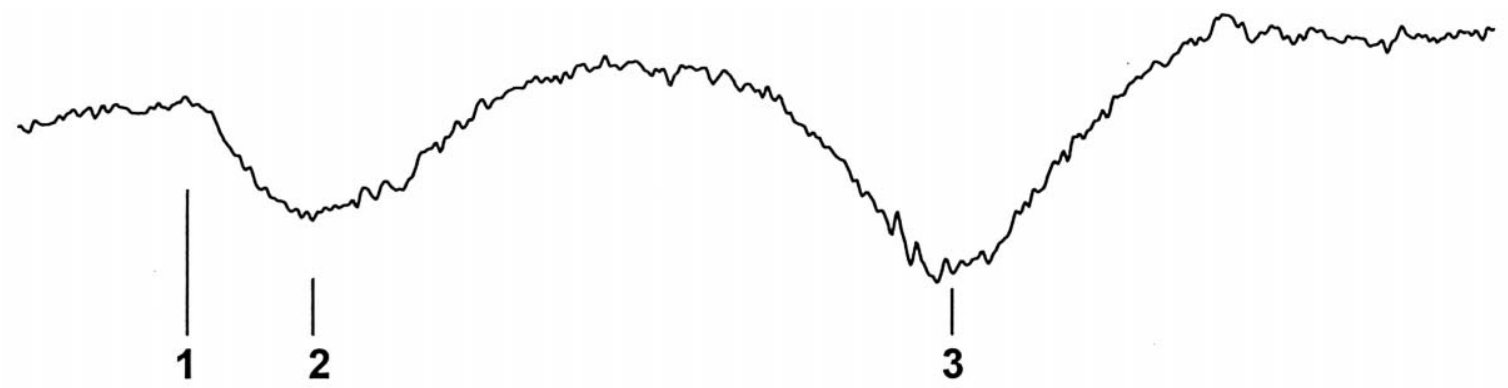

1. $T=45.5 \mathrm{msec}$

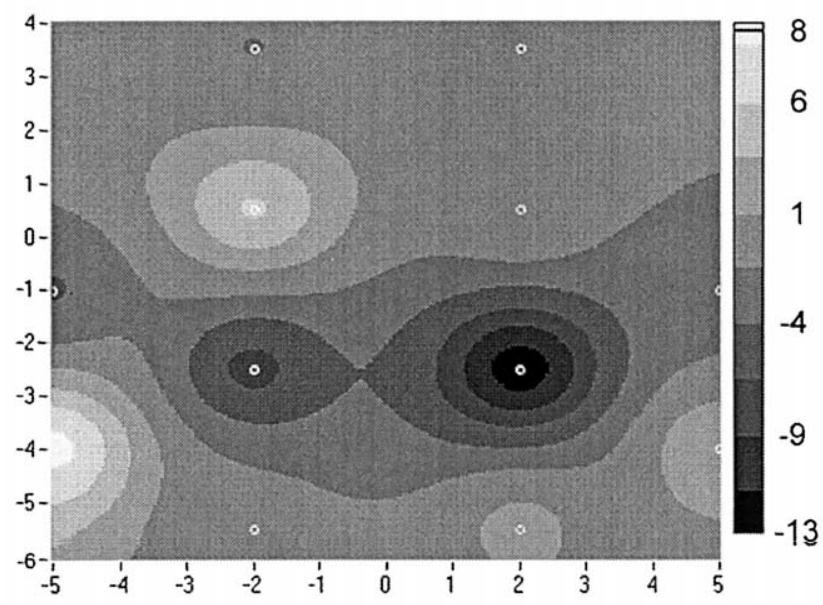

2. $T=78 \mathrm{msec}$

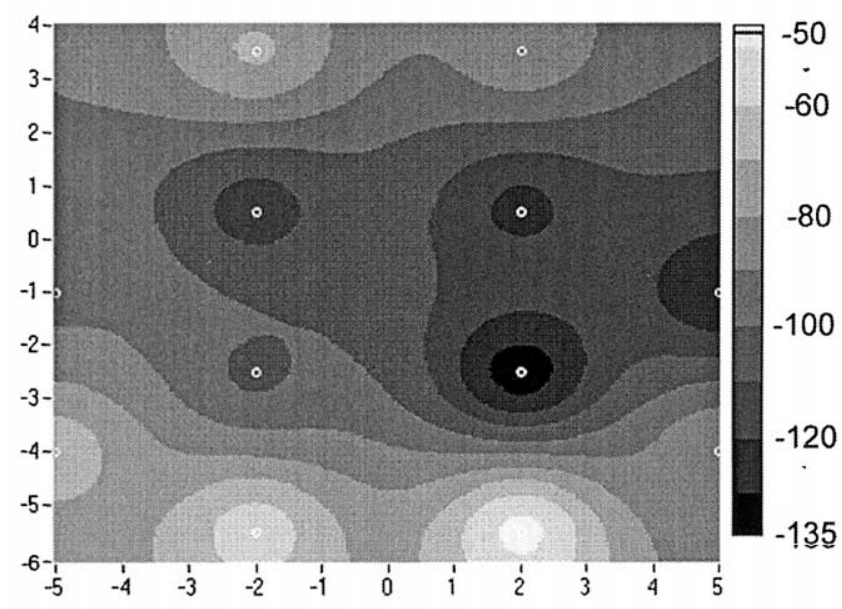

3. $\mathrm{T}=240.5 \mathrm{msec}$

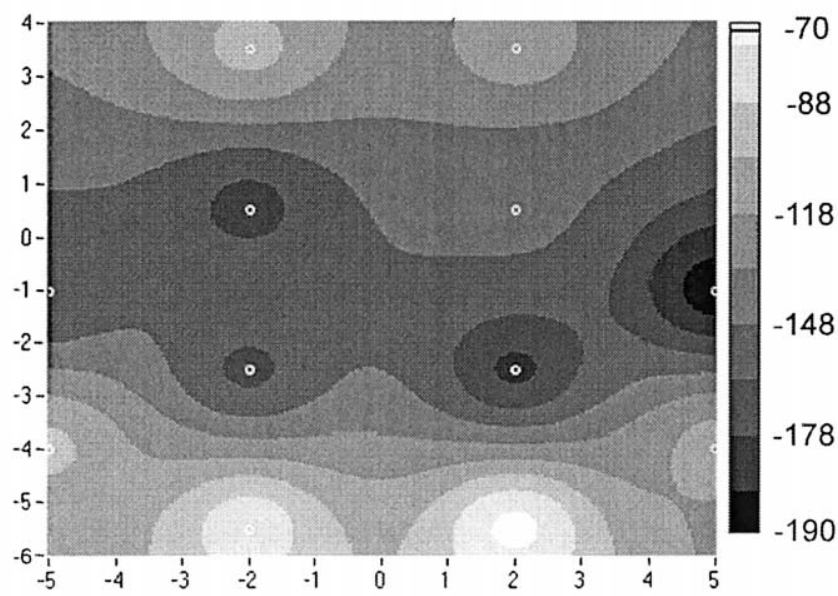

Fig. 4. Topographical map of the laser-evoked potentials. The upper trace is the laser-evoked potential at channel 5 of Fig. 3 . Three isopotential maps are made at latencies $45.5,78$, and $240.5 \mathrm{~ms}$ from the start of the $\mathrm{CO}_{2}$-laser stimulation point respectively. Note a localized sink $(T=45.5$ $\mathrm{ms}$ ) followed by a widespread distributed component $(T=78 \mathrm{~ms})$. A widespread potential distribution was also found for the second negative component $(T=240.5 \mathrm{~ms})$. The units in the grey scale are expressed in $\mu \mathrm{V}$. The zero point of the topographical maps is at bregma. The units of the horizontal and vertical scales are in millimeters $(\mathrm{mm})$. 

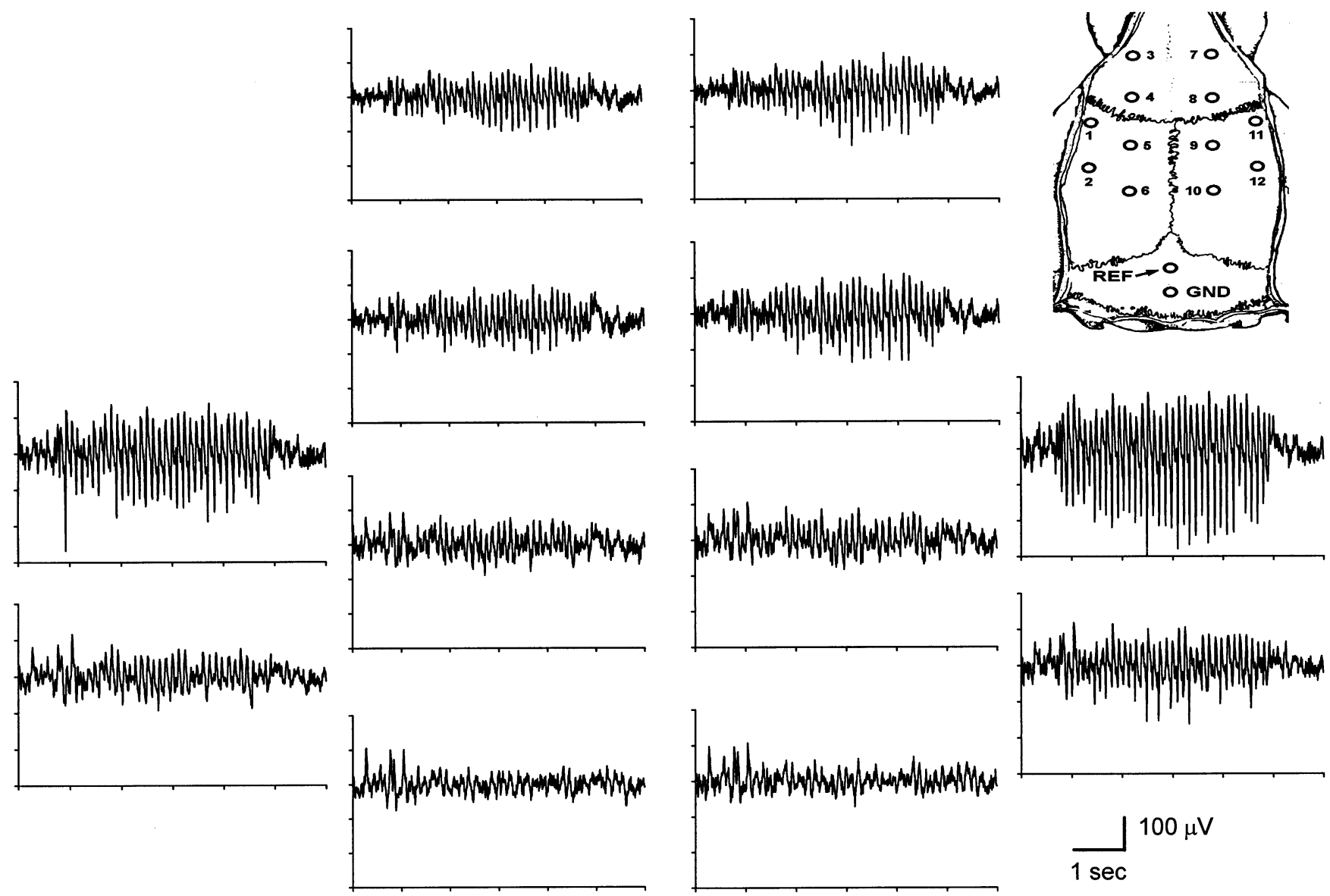

Fig. 5. Simultaneously recorded 12 monopolar EEG traces in one episode of sleep-spindle rhythm. An example of 12-channel recordings of cortical field potentials displayed at relative recording sites over the skull (indicated in inset).

In the analytic procedures, we took into account the influence of common reference in spatio-temporal analysis. Ideally, the common reference lead should be inactive. However, the reference lead was not inactive in reality. Therefore, the specific characteristics (such as correlation coefficient, coherence and phase) between monopolar recording data trials were influenced by the common reference lead (Fein et al., 1988, see results below). In an attempt to alleviate this problem, three reference-free methods (Wilson, local average and weighted average methods) were implemented. These methods, which essentially perform a spatial high-pass filtering function, were tested for their usefulness in improving the detection of near-field cortical activity by filtering out widespread far-field subcortical potentials. In implanting the reference methods, the reference signal (i.e. widespread signal) must be calculated first, and then this reference signal subtracted from each individual channel to obtain the near-field potentials. Different derivations used different weighting factors for the calculation of the reference signal. In the Wilson method, the weighting factors were obtained by the inverse of the number of the active electrodes except the central electrode (Osselton, 1965). In the local average method, the weighting factors were calculated by the inverse linear distance from the local contiguous electrodes with the constraint of unitary sum (Hjorth, 1980). The weighting factors in the weighted average method (Lemos and Fisch, 1991) were determined by first calculating the inverse distances between each central, nonreference electrode and each of its remaining reference electrodes. The weighting factor for a particular reference electrode was then calculated by dividing the inverse of its distance by the sum of all the inverse distances between the central, non-reference electrode and each of the remaining electrodes.

\section{Performance and discussion}

The impedances of 30 randomly selected screw electrodes, measured from the voltage drop of a $100 \mathrm{~Hz}, 10$ $\mathrm{mV}$ sinusoidal wave source passing through a seriallyconnected $10 \mathrm{M} \Omega$ resistor, varied from 1.5 to $10 \mathrm{k} \Omega$ (mean $7.5 \mathrm{k} \Omega$ ). To avoid distortions in the field potential recording, the input impedance of the preamplifier was at least 1000 times greater than the impedance of the recording electrodes. The input impedance (10 G $\Omega$ ) 
of the instrumentation amplifier of our self-designed amplifier fulfilled this requirement.

Testing of the 16-channel amplifier system in an unshielded environment showed excellent performance. In this system, the frequencies of half amplitude were at 0.3 and $500 \mathrm{~Hz}$, and flat bandpass was between 1 and $300 \mathrm{~Hz}$. The precision of the gain setting was kept within $2 \%$. The variation of gain among channels was kept within $0.2 \%$. The cross-talk rejection ratio between channels was greater than $80 \mathrm{~dB}$. The performance of our multichannel amplifier system was compared with that of the P511 amplifier (Grass Instruments). Two original data segments, each from our amplifier assembly $(2000 \times, 0.3-500 \mathrm{~Hz})$ and Grass P511 $(2000 \times$, $0.3-300 \mathrm{~Hz}$ ) are shown in the lower part of Fig. 1. The noise of our amplifier was about $1.5 \mu \mathrm{V}_{\mathrm{p}-\mathrm{p}}$ and half to one-third of that of the Grass amplifier. The cost of the whole amplifier assembly (including power system) was below US\$300. Furthermore, its size and the weight was much less than Grass P511 assembly. The entire recording system could be placed near the animal to reduce interference. The results described above demonstrated the good performance of our multichannel amplifier system.

Short pulses of $\mathrm{CO}_{2}$-laser radiation have been reported to activate mainly $\mathrm{A} \delta$ and $\mathrm{C}$ nociceptors in rats (Devor et al., 1982) and humans (Bromm et al., 1984),

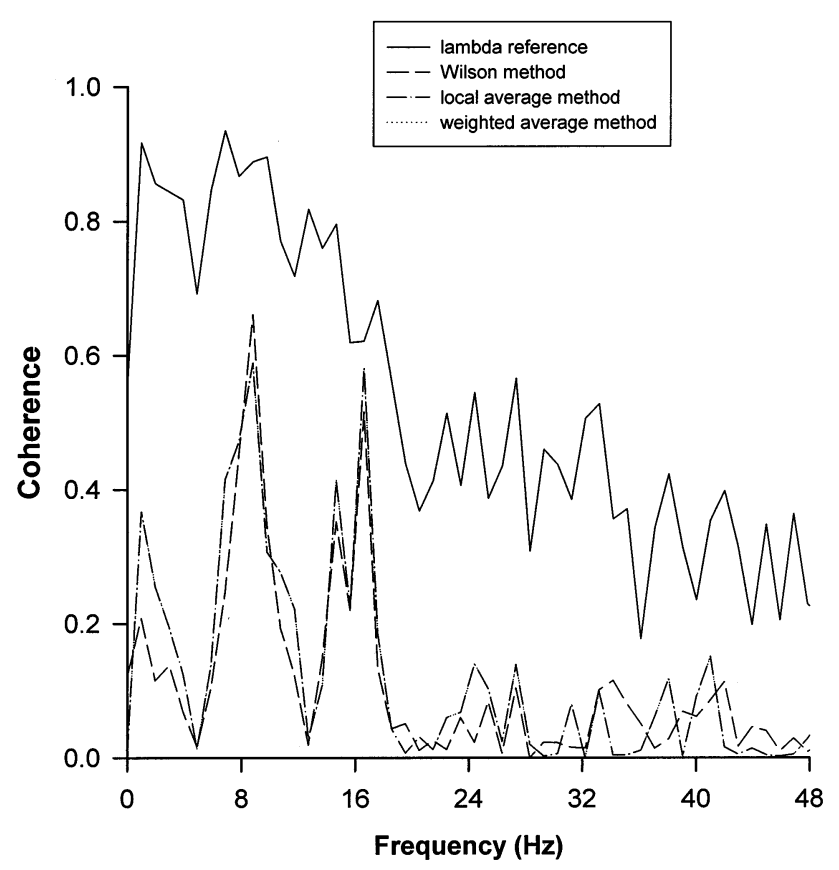

Fig. 6. Coherence of EEG segments between channels 5 and 9 in four reference conditions. Forty EEG segments with 1-s durations were selected. High coherence was present in the low-frequency range at the lambda reference, as shown (solid line). Two distinct frequency bands with high coherence are depicted using reference-free methods. The results of local average and weighted average methods are almost the same. without activating low-threshold mechanoreceptors. An example of the evoked responses to $\mathrm{CO}_{2}$-laser stimuli is illustrated here. The laser-evoked potentials before and after i.p. sodium pentobarbital injection $(50 \mathrm{mg} / \mathrm{kg})$ are shown in Fig. 2. In the conscious stage, two clearly distinctive components (marked by arrow and arrowhead, respectively) were observed after middle tail stimulation, and the delays of these two components corresponded to the conduction velocity of $\mathrm{A} \delta$ and $\mathrm{C}$ afferent fibers, respectively (Shaw et al., 1997). Both components disappeared after anesthesia within the first $60 \mathrm{~min}$, and the rat did not show any behavioral response to $\mathrm{CO}_{2}$-laser stimulation. Two hours after pentobarbital injection, the waveform of the $\mathrm{C}$-fiber component reappeared with a polarity change. The rats showed signs of consciousness (eye blinking, whisker movement, muscle twitching, crawling sometimes), and its tail flicked in response to $\mathrm{CO}_{2}$-laser stimulation. Three hours later, the C-fiber component was restored but with lower magnitude. The rats could move slowly at this time. The first component was more sensitive to anesthesia. It did not recover during the 3-h observation period after pentobarbital administration. We believe that this is a reason why this component has not been consistently observed in halothane-anesthetized rats as reported by Kalliomaki et al. (1993). Furthermore, the negative polarity of both components in the conscious rats is the same as reported in humans (Bromm and Treede, 1987). Our present results also confirm that anesthetic strongly modifies the nociceptive processing of information (Kochs et al., 1990; Vogt, 1991).

The spatial relationship between the different cortical areas was then depicted in a grey-scale diagram. An example of the laser EP response to tail base stimulation is shown in Fig. 3. Three latencies relative to $\mathrm{CO}_{2}$-laser stimulation are illustrated as topographical maps (Fig. 4). A bilateral concentric sink was initially generated at SI (latency, $45.5 \mathrm{~ms}$ ). The cortical potential (latency, $78 \mathrm{~ms}$ ) was found to occupy a rather large cortical area (mainly within the somatomotor cortex). For the second component, no concentric current portrait was observed, and widespread potential distribution was also seen (latency, $240.5 \mathrm{~ms}$ ). From spatial mapping, SI seemed to participate in nociceptive information processing. Furthermore, we found that SI was the first activated cortical area for nociceptive afferent fibers and that displayed a localized potential.

Since raw EEG/EP epochs with lambda reference were obtained at the beginning, one could use these to calculate reference-free trials. Fig. 5 depicts sleep spindle activity $(7-14 \mathrm{~Hz})$ recorded in the lambda reference condition. The traces of EEG recordings in lambda reference were very similar. The correlation coefficients $(r)$ between channel 5 (somatosensory lead) and the other channels under four different conditions were 
Table 1

The correlation coefficients of the EEG segments $(1 \mathrm{~s})$ vs. channel 5 are presented in four reference conditions ${ }^{\mathrm{a}}$

\begin{tabular}{|c|c|c|c|c|c|c|c|c|c|c|c|}
\hline & $\mathrm{CH} 1$ & $\mathrm{CH} 2$ & $\mathrm{CH} 3$ & $\mathrm{CH} 4$ & $\mathrm{CH} 6$ & $\mathrm{CH} 7$ & $\mathrm{CH} 8$ & $\mathrm{CH} 9$ & $\mathrm{CH} 10$ & $\mathrm{CH} 11$ & $\mathrm{CH} 12$ \\
\hline Lambda & $0.7 \pm 0.08$ & $0.89 \pm 0.04$ & $0.69 \pm 0.12$ & $0.85 \pm 0.06$ & $0.7 \pm 0.1$ & $0.69 \pm 0.1$ & $0.86 \pm 0.1$ & $0.63 \pm 0.14$ & $0.5 \pm 0.1$ & $0.71 \pm 0.09$ & $0.73 \pm 0.08$ \\
\hline Wilson & $-0.16 \pm 0.15$ & $-0.06 \pm 0.2$ & $0.18 \pm 0.2$ & $0.22 \pm 0.2$ & $-0.23 \pm 0.14$ & $-0.35 \pm 0.2$ & $0.31 \pm 0.2$ & $0.16 \pm 0.19$ & $-0.11 \pm 0.13$ & $-0.1 \pm 0.16$ & $0.1 \pm 0.16$ \\
\hline Local average & $-0.23 \pm 0.13$ & $0.11 \pm 0.31$ & $0.34 \pm 0.17$ & $0 \pm 0.16$ & $0.06 \pm 0.18$ & $-0.31 \pm 0.15$ & $0.41 \pm 0.18$ & $0.04 \pm 0.17$ & $-0.16 \pm 0.11$ & $0.03 \pm 0.14$ & $0.12 \pm 0.16$ \\
\hline Weighted aver- & $-0.23 \pm 0.13$ & $0.11 \pm 0.31$ & $0.34 \pm 0.17$ & $0 \pm 0.16$ & $0.06 \pm 0.18$ & $-0.31 \pm 0.15$ & $0.41 \pm 0.18$ & $0.04 \pm 0.17$ & $-0.16 \pm 0.11$ & $0.03 \pm 0.14$ & $0.12 \pm 0.16$ \\
\hline
\end{tabular}
age

${ }^{\text {a }}$ Data are presented as mean $\pm \mathrm{SD}$. 
calculated in 1-s EEG segments. The $r$-values (mean \pm SD) are shown in Table 1. Using the lambda reference, the EEG trial of channel 5 was very similar to the other channels (pooled $r, 0.72 \pm 0.09$ ). However, $r$-values were lower in all the reference-free derivations. Coherence can be used to evaluate the relationship between two different cortical areas. We calculated the coherence between channel 5 and channel 9 under four different conditions during the sleep-spindle period. The coherence value of the lambda-reference condition is high in the low-frequency range (Fig. 6, solid line). High coherence values within wide bandwidth induced by the common reference lead was noted, confirming previous reports (French and Beaumont, 1984; Fein et al., 1988). The high coherence values in the low-frequency range, however, were not present in all reference-free derivations. Moreover, two distinct peaks ( $\alpha$ band and $\beta 1$ band) appeared in the reference-free derivations (Fig. 6). The first peak was located within the bandwidth of the sleep-spindle rhythm, and as such, might be generated by the corticothalamic and corticocortical network (Contreras et al., 1996). The other coupled peak was in the range of the $\beta 1$ bandwidth $(12-20 \mathrm{~Hz})$ which is a behavioral-related bandwidth (French and Beaumont, 1984). These results therefore demonstrated the advantage of using reference-free signal derivations to minimize the influence of both common reference and widespread signals in EEG analysis.

In summary, a technique has been developed to record from different brain sites in freely moving rats. A low noise and low cost miniature 16-channel amplifier was developed. This amplifier enables one to obtain artifact-free multisite recordings in freely moving rats. An on-line interactive icon-based analysis program was also developed. It provides the reference-free derivation to reduce the influence from the common reference electrode. This compact recording system allowed us to study the cortical distribution of specific patterns of the EEG/EP due to different external stimuli and corticocortical interactions in conscious animal.

\section{Acknowledgements}

We thank Professors JK Chapin and RCS Lin and Dr KM Simpson, Allegheny University, USA, for their comments on the manuscript. The present study was supported by grants NSC84-2311-B002-027 and NSC85-2311-B002-031 from the National Science Council of the Republic of China (Taiwan).

\section{References}

Bromm B, Treede RD. Human cerebral potentials evoked by $\mathrm{CO}_{2}$ laser stimuli causing pain. Exp Brain Res 1987;67:153-62.
Bromm B, Jahnke MT, Treede RD. Responses of human cutaneous afferents to $\mathrm{CO}_{2}$-laser stimuli causing pain. Exp Brain Res 1984;55:158-66.

Chapin JK, Lin CS. The somatic sensory cortex of the rat. In: Kolb B, Tees RC, editors. The Cerebral Cortex of the Rat. Cambridge, MA: MIT Press, 1990:341-80.

Contreras D, Destexhe A, Sejnowski TJ, Steriade M. Control of spatiotemporal coherence of a thalamic oscillation by corticothalamic feedback. Science 1996;274:771-4.

Devor M, Carmon A, Forstig R. Primary afferent and spinal sensory neurons that respond to brief pulses of intense infrared laser radiation: a preliminary survey in rats. Exp Neurol 1982;76:48394.

Dong WK, Salonen LD, Kawakami Y, Shiwaku T, Kaukoranta M, Martin RF. Nociceptive responses of trigeminal neurons in SII-7b cortex of awake monkey. Brain Res 1989;484:314-24.

Fein G, Raz J, Brown FF, Merrin EL. Common reference coherence data are confounded by power and phase effects. Electroencephalogr Clin Neurophysiol 1988;69:581-4.

French CC, Beaumont JG. A critical review of EEG coherence studies of hemisphere function. Int J Psychophysiol 1984;1:24154.

Head H, Holmes G. Sensory disturbances from cerebral lesions. Brain 1911;34:102-254.

Hjorth B. Source derivation simplifies topographical EEG interpolation. Am J EEG Technol 1980;20:121-32.

Jones AK, Brown WD, Friston KJ, Qi LY, Frackowiak RS. Cortical and subcortical localization of response to pain in man using positron emission tomography. Proc R Soc Lond B 1991;244:3944.

Kalliomaki J, Weng HR, Nilsson HJ, Schouenborg J. Nociceptive C fibre input to the primary somatosensory cortex (SI). A field potential study in the rat. Brain Res 1993;622:262-70.

Kenshalo DR, Isensee O. Responses of primate SI cortical neurons to noxious stimuli. J Neurophysiol 1983;50:1479-96.

Kenshalo DR, Willis WD. The role of the cerebral cortex in pain system. In: Peter A, Jones EG, editors. Cerebral Cortex: Normal and Altered States of Function, vol. 9. New York: Plenum, 1991:153-212.

Kenshalo DR, Chudler EH, Anton F, Dubner R. SI nociceptive neurons participate in the encoding process by which monkey perceive the intensity of noxious thermal stimulation. Brain Res 1988;454:378-82.

Kitamura Y, Kakigi R, Hoshiyama M, Koyama S, Shimojo M, Watanabe S. Pain-related somatosensory evoked magnetic fields. Electroencephalogr Clin Neurophysiol 1995;95:463-74.

Kochs E, Treede RD, Schulte am Esch J, Bromm B. Modulation of pain-related somatosensory evoked potentials by general anesthesia. Anesth Analg 1990;71:225-30.

Lamour Y, Guilbaud G, Willer JC. Rat somatosensory (SmI) cortex: II. Laminar and columnar organization of noxious and non-noxious inputs. Exp Brain Res 1983a;49:46-54.

Lamour Y, Willer JC, Guilbaud G. Rat somatosensory (SmI) cortex: I. Characteristics of neuronal responses to noxious stimulation and comparison with responses to non-noxious stimulation. Exp Brain Res 1983b;49:35-45.

Lemos MS, Fisch BJ. The weighted average reference montage. Electroencephalogr Clin Neurophysiol 1991;79:361-70.

Luders H, Lesser RP, Dinner DS, Morris HH. Optimizing stimulating and recording parameters in somatosensory evoked potential studies. J Clin Neurophysiol 1985;2:383-96.

Matsumoto N, Sato T, Suzuki TA. Characteristics of the tooth pulp-driven neurons in a functional column of the cat somatosensory cortex (SI). Exp Brain Res 1988;74:263-71.

Oppenheim AV, Schafer RW. Discrete-Time Signal Processing, 2nd edn. Englewood Cliffs, NJ: Prentice-Hall, 1989. 
Osselton JW. Acquisition of EEG data by bipolar, unipolar and average reference methods: a theoretical comparison. Electroencephalogr Clin Neurophysiol 1965;19:527-8.

Perrin F, Pernier J, Bertrand O, Giard MH, Echallier JF. Mapping of scalp potentials by surface spline interpolation. Electroencephalogr Clin Neurophysiol 1987;66:75-81.

Schouenborg J, Kalliomaki J, Gustavsson P, Rosen I. Field potentials evoked in rat somatosensory cortex (SI) by impulses in cutaneous A $\beta$ - and C-fibres. Brain Res 1986;397:86-92.

Shaw FZ, Chen RF, Lee EHY, Tsao HW, Yen CT. Mapping of cortical field potentials evoked by electrical, mechanical and laser heat stimulation of the rat tail. Soc Neurosci Abstr 1997;23:440.

Talbot JD, Marrett S, Evans AC, Meyer E, Bushnell MC, Duncan GH. Multiple representations of pain in human cerebral cortex. Science 1991;251:1355-8.

Vogt BA. The role of layer I in cortical function. In: Peter A, Jones EG, editors. Cerebral Cortex: Normal and Altered States of Function, vol. 9. New York: Plenum, 1991:49-80.

Yen CT, Huang $\mathrm{CH}$, Fu SE. Surface temperature change, cortical evoked potential and pain behavior elicited by $\mathrm{CO}_{2}$ lasers. Chin J Physiol 1994;37:193-9. 